\title{
Quetiapine monotherapy for bipolar depression
}

\author{
Michael E Thase \\ Departments of Psychiatry, University \\ of Pennsylvania School of Medicine, \\ Philadelphia, PA, USA; the Philadelphia \\ Veterans Affairs Medical Center, \\ Philadelphia, PA, USA; and the \\ University of Pittsburgh Medical \\ Center, Pittsburgh, PA, USA
}

\begin{abstract}
Bipolar depression is more common, disabling, and difficult-to-treat than the manic and hypomanic phases that define bipolar disorder. Unlike the treatment of so-called "unipolar" depressions, antidepressants generally are not indicated as monotherapies for bipolar depressions and recent studies suggest that -even when used in combination with traditional mood stabilizers - antidepressants may have questionable value for bipolar depression. The current practice is that mood stabilizers are initiated first as monotherapies; however, the antidepressant efficacy of lithium and valproate is modest at best. Within this context the role of atypical antipsychotics is being evaluated. The combination of olanzapine and the antidepressant fluoxetine was the first treatment to receive regulatory approval in the US specifically for bipolar I depression. Quetiapine was the second medication to be approved for this indication, largely as the result of two pivotal trials known by the acronyms of BOLDER (BipOLar DEpRession) I and II. Both studies demonstrated that two doses of quetiapine (300 mg and $600 \mathrm{mg}$ given once daily at bedtime) were significantly more effective than placebo, with no increased risk of patients switching into mania. Pooling the two studies, quetiapine was effective for both bipolar I and bipolar II depressions and for patients with (and without) a history of rapid cycling. The two doses were comparably effective in both studies. Although the efficacy of quetiapine monotherapy has been established, much additional research is necessary. Further studies are needed to more fully investigate dose-response relationships and comparing quetiapine monotherapy to other mood stabilizers (lithium, valproate, and lamotrigine) in bipolar depression, both singly and in combination. Head-to-head studies are needed comparing quetiapine to the olanzapinefluoxetine combination. Longer-term studies are needed to confirm the persistence of response and to better gauge effects on metabolic profiles across months of therapy. A prospective study of patients specifically seeking treatment for rapid cycling and those with a history of treatment-emergent affective shifts also is needed. Despite the caveats, as treatment guidelines are revised to incorporate new data, the efficacy and tolerability of quetiapine monotherapy must be given serious consideration.
\end{abstract}

Keywords: bipolar disorder, manic depression, depression, quetiapine, mood stabilizer

\section{Introduction}

Bipolar disorder is a highly recurrent and not infrequently chronic illness that is recognized as one of the world's 10 greatest public health problems (Murray and Lopez 1997). For the majority of patients, the periods of depression far exceed those of mania, in terms of both frequency and duration (Post et al 2003; Judd et al 2002, 2003). For individuals with bipolar I disorder, for example, days spent with depressive symptoms are about three times more common than days spent with hypomanic or manic symptoms (Judd et al 2002). The dominance of the depressed pole of the illness is even more dramatic individuals with bipolar II disorder: in one prospective study conducted across nearly 13 years, patients with bipolar II disorder spent almost 40 times the days with depressive symptoms as compared to the days spent with hypomanic symptoms (Judd et al 2003).

Despite the dramatic and life-disrupting nature of mania, recent studies have also documented that it is the more long-lasting depressive episodes that have the greater 
deleterious effects on quality of life and functionality (Judd et al 2005; Depp et al 2006). The burden imposed by bipolar depression on the family and loved ones exceeds that of bipolar mania or unipolar depression, perhaps all the more remarkable in view of the greater risk of psychosis, violent behaviour, and increased frequency of hospitalization associated with mania (Post 2005; Hirschfeld 2004). The perceived stigma of the condition may also add to the burden placed on the family or primary caregiver (Perlick et al 2004). The assessment of caregiver burden is further impeded by the unique characteristics of bipolar depression - including the unfortunate tendency for milder episodes to go unrecognized or untreated and the high incidence of subsyndromal inter-episode symptoms (Ogilvie et al 2005). Perhaps not surprisingly, the depressive episodes also are more directly linked to reduced longevity in bipolar disorder, particularly through suicide but perhaps also to increased risks of obesity and cardiovascular disease (Dilsaver et al 1997; Fagiolini et al 2002; Mitchell and Malhi 2004).

Despite the obvious clinical importance of the depressed phase of bipolar disorder, remarkably few controlled studies of first- and second-line treatments have been performed (Thase 2005). The paucity of well-designed studies essentially precludes the practice of evidence-based medicine and for some important questions (eg, "If an antidepressant is used and appears to be effective, how long should it be maintained?") there is not consensus about best practices, which no doubt hampers clinical decision-making (Thase 2005; Ostacher 2006). Indeed, in the largest placebo-controlled study of the role of antidepressants in bipolar depression conducted to date, the addition of paroxetine or bupropion to optimized therapy with mood stabilizers resulted in no added benefit as compared to therapy with mood stabilizers alone (Sachs et al 2007). For the prescribing physician, the need to swiftly deliver effective pharmacotherapy to lessen suffering and minimize functional impairments is paramount, and appears to foster the continued use of antidepressants in bipolar depression despite the lack of clear-cut evidence that they improve outcomes. Nevertheless, the decision to initiate therapy with an antidepressant to hasten recovery is not without attendant risks, including treatment-emergent affective switches (TEAS) or acceleration of cycling and, as a result, the ranking of antidepressants in contemporary practice guidelines continues to drop in favor of other strategies (Thase 2005; Yatham et al 2006).

Many expert panels recommend initiating mood stabilizers alone, ie, before considering whether or not an antidepressant is indicated. If one accepts the validity of the "mood stabilizer first" strategy, then lithium and three anticonvulsants (valproate, carbamazepine, and lamotrigine) might be nominated as candidates for first line of therapy for bipolar depression (Thase 2005; Grunze 2005). However, none of these medications is renowned for having powerful antidepressant effects (Thase 2005) and - primarily for reasons of tolerability and safety - few clinicians would use carbamazepine as the first step in a treatment algorithm. Even lithium salts, which arguably have the best evidence of efficacy from placebo-controlled studies (Zornberg and Pope 1993; Thase and Sachs 2000), do not exert particularly robust antidepressant effects (Thase 2005). The search for an effective monotherapy for bipolar depression thus goes on.

Emerging data suggest that the list of medications that are classified as mood stabilizers eventually may need to be expanded to include the class of medications known as atypical antipsychotics. All five of the more widely prescribed atypical antipsychotics (in alphabetical order: aripiprazole, olanzapine, quetiapine, risperidone, and ziprasidone) have established antimanic efficacy. Consistent with proposed criteria to define mood stabilizers (see, for example, Ketter and Calabrese 2002; Goodwin and Malhi 2007), atypical antipsychotics are unlikely to cause TEAS and two members of the class (olanzapine and aripiprazole) have received a formal indication for prophylaxis against manic relapse following successful acute therapy. Starting with observations from studies that included patients with mixed manic states, there is slowly increasing evidence to indicate that atypical antipsychotics also have antidepressant effects (Keck 2005; Nemeroff 2005). In fact, the first treatment to be approved by the United States Food and Drug Administration (FDA) specifically for bipolar depression is the proprietary combination of olanzapine and the selective serotonin reuptake inhibitor (SSRI), fluoxetine. In the pivotal trials that led to that indication, olanzapine monotherapy was also studied and found to have intermediate efficacy: greater than placebo but significantly less than the olanzapine-fluoxetine combination (OFC) (Tohen et al 2003).

This review will focus on the second atypical antipsychotic to be systematically studied as a monotherapy for bipolar depression, quetiapine. The results of the research program that led to the FDA approval of quetiapine monotherapy for bipolar depression will be summarized in detail. Quetiapine, which is the first-and currently only-monotherapy approved by the FDA to treat both the depressive and manic episodes associated with bipolar disorder, has been ranked as a first-line treatment of bipolar depression in the recently 
updated treatment guidelines published by the Canadian Network for Mood and Anxiety Treatments (CANMAT) (Yatham et al 2006).

\section{Efficacy against depressive symptoms}

Regulatory approval of quetiapine monotherapy for bipolar depression was primarily based on two similar randomized controlled trials (RCTs) known by the acronyms BOLDER (BipOLar DEpRession) I and II. Both of these 8-week, placebo-controlled, double-blind studies compared two doses of quetiapine - $300 \mathrm{mg}$ per day and $600 \mathrm{mg}$ per day. Both studies used once daily dosing (at bedtime) and the same rapid titration schedule, with maximum study dose achieved by the 8th day of treatment. Both studies included patients with bipolar I and bipolar II depressive episodes and allowed otherwise eligible patients with histories of rapid cycling to enroll. Both studies used change in the Montgomery-Åsberg Depression Rating Scale (MADRS) total score as the primary endpoint. Together, the BOLDER I (Calabrese et al 2005) and BOLDER II (Thase et al 2006) studies represent the largest placebo-controlled data set to date that includes patients with bipolar I and bipolar II depressions.

BOLDER I enrolled 542 patients meeting DSM-IV criteria for a current episode of bipolar I or bipolar II depression, according to DSM-IV criteria (Calabrese et al 2005). In order to enter the study, outpatients had to score at least 20 on the 17-item Hamilton Depression Scale (HAM-D17), as well as have a score of at least 2 on HAM-D item 1 (depressed mood). Pretreatment MADRS scores indicated that the unmedicated study group presented with moderate-to-severe levels of depressive symptoms (see, for example, Muller et al 2003).

Both doses of quetiapine resulted in significant improvements in MADRS total scores at all time points measured, with statistical significance over placebo detected after only 1 week of treatment (the first assessment point of the study) and maintained at every time point thereafter (see Figure 1a). The proportion of patients classified as responders to treatment, defined as a $\geq 50 \%$ improvement in MADRS total score at study endpoint (using the "last observation carried forward [LOCF] convention" to estimate the final scores of study dropouts) was significantly higher in both groups receiving active quetiapine (58\% in both groups) than in the group randomized to placebo (36\%). Remission rates (defined as a final MADRS total score $\leq 12$ ) followed a similar pattern (53\% for both $300 \mathrm{mg}$ and $600 \mathrm{mg}$ quetiapine, 28\% for placebo). Individuals treated with either dose of quetiapine were faster to respond to treatment and to achieve remission than those receiving placebo (median time to response was 22 days for both doses of quetiapine versus 36 days for placebo, and median times to remission were 29, 27, and 65 days for $300 \mathrm{mg}$ quetiapine, $600 \mathrm{mg}$ quetiapine, and placebo, respectively).

The results of the BOLDER II trial $(n=509)$ fully replicated the first study in terms of the primary outcome variable, with quetiapine-treated patients displaying significantly greater mean improvement in MADRS total scores than placebo-treated patients at all time points from Week 1 onward (Figure 1b) (Thase et al 2006). Response rates for both doses of quetiapine monotherapy were also similar to those observed in the original study after 8 weeks of treatment (60\%, 58\%, and $45 \%$ for the $300 \mathrm{mg}, 600 \mathrm{mg}$, and placebo groups, respectively), as were remission rates ( $52 \%$ for both groups receiving active quetiapine as compared to $37 \%$ for the group receiving placebo). Looking across the two studies, the only appreciable difference was the higher placebo response/remission rates observed in BOLDER II, which could possibly be attributable to increased expectations from physicians and patients alike, in light of the positive findings arising from BOLDER I.

In both BOLDER studies, improvements on the secondary rater-administered measure, the HAM-D ${ }_{17}$, mirrored those reported on the MADRS scale. For example, both groups receiving active quetiapine again experienced significantly greater mean improvements from Week 1 onward compared with the group receiving placebo.

With respect to the impact of quetiapine on specific depressive symptoms, at study endpoint improvements were detected in nine of the 10 individual items in BOLDER I, and in nine individual items in BOLDER II. Figure 2 summarizes improvements in individual items of the MADRS scale in the BOLDER studies. It is important to note that significant improvements were observed on the core symptoms of depression, including apparent sadness, reported sadness, suicidal thoughts, and pessimistic thoughts, in addition to improvements in sleep and anxiety.

\section{Efficacy in patient subgroups}

Since the patient populations enrolled in the BOLDER studies included individuals with both bipolar I and bipolar II depression, and those with and without a rapid-cycling disease course, the results of the BOLDER trials were examined to determine if quetiapine was particularly effective - or ineffective - in various patient subgroups. Although there are important differences between bipolar I and bipolar II disorders (as well as between patients who meet criteria for 
Study Week

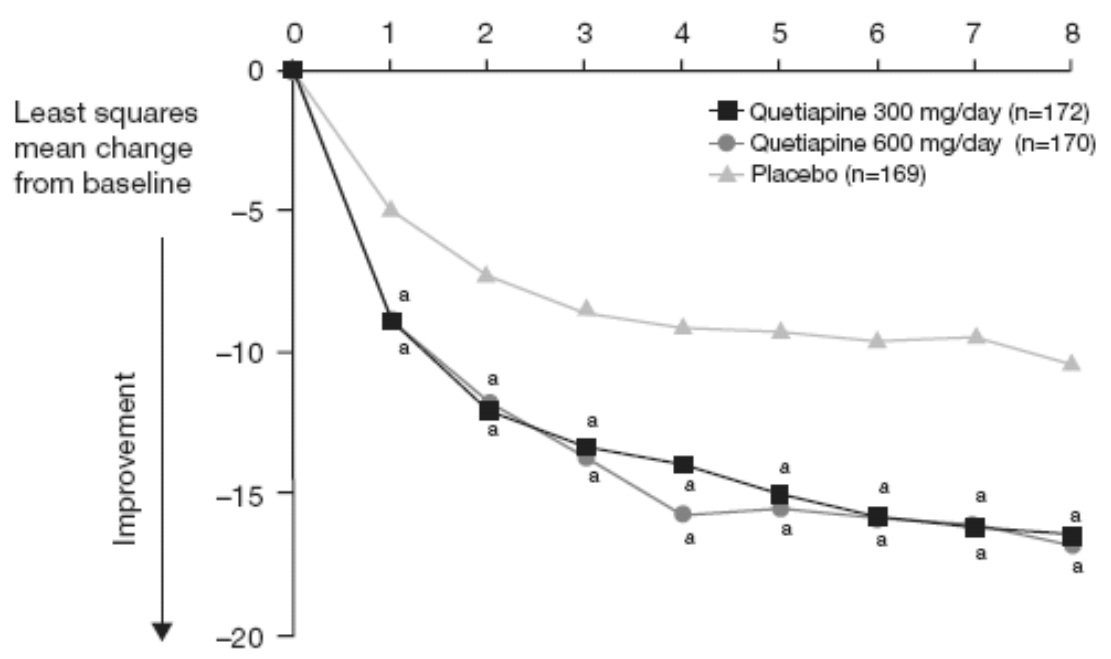

${ }^{a} \mathrm{p}<0.001$ vs placebo

Figure Ia Least-squares mean change from baseline in Montgomery-Åsberg Depression Rating Scale (MADRS) total score at each assessment of outpatients with bipolar I or II disorder who experienced a major depressive episode (BOLDER I).

rapid cycling and those who do not) (Yatham et al 2005), demonstration that a novel treatment is comparably effective across the subgroups could greatly simplify clinical management. The combined BOLDER data set shows that both bipolar I and bipolar II patient groups exhibited significant improvements in MADRS total score following treatment with either dose of quetiapine (300 mg per day or $600 \mathrm{mg}$ per day) compared with placebo (Figure 3).

Study Week

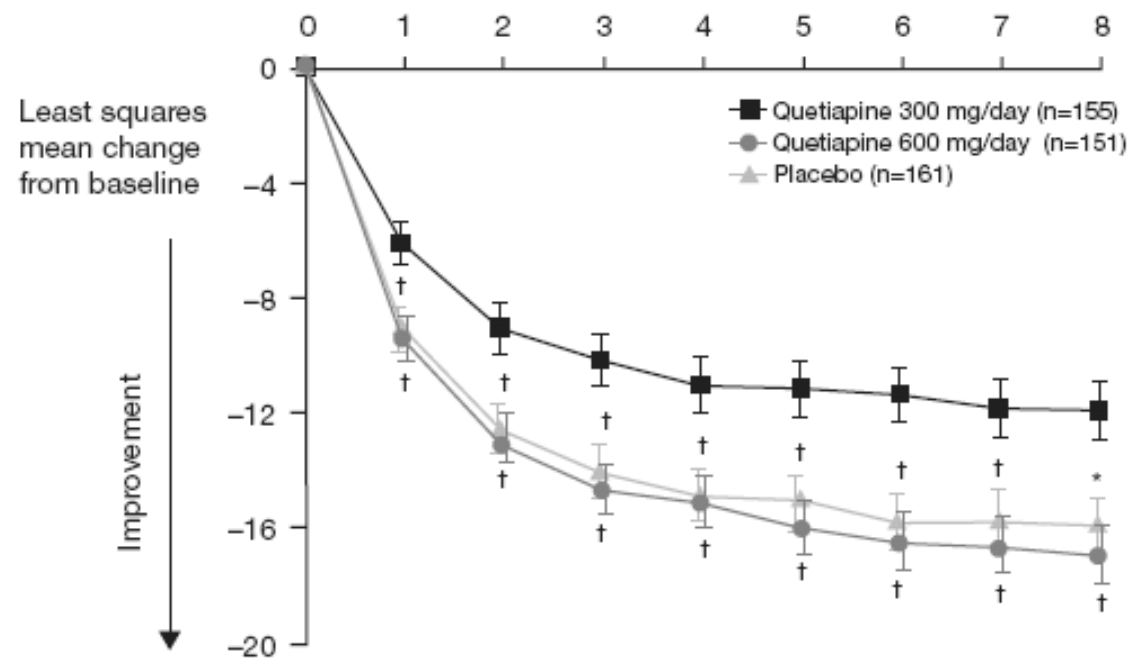

${ }^{*} p<0.01,{ }^{\dagger} p<0.001$ vs placebo

Figure I b Least-squares mean change from baseline in Montgomery-Åsberg Depression Rating Scale (MADRS) total score at each assessment of outpatients with bipolar I or II disorder who experienced a major depressive episode (BOLDER II). 


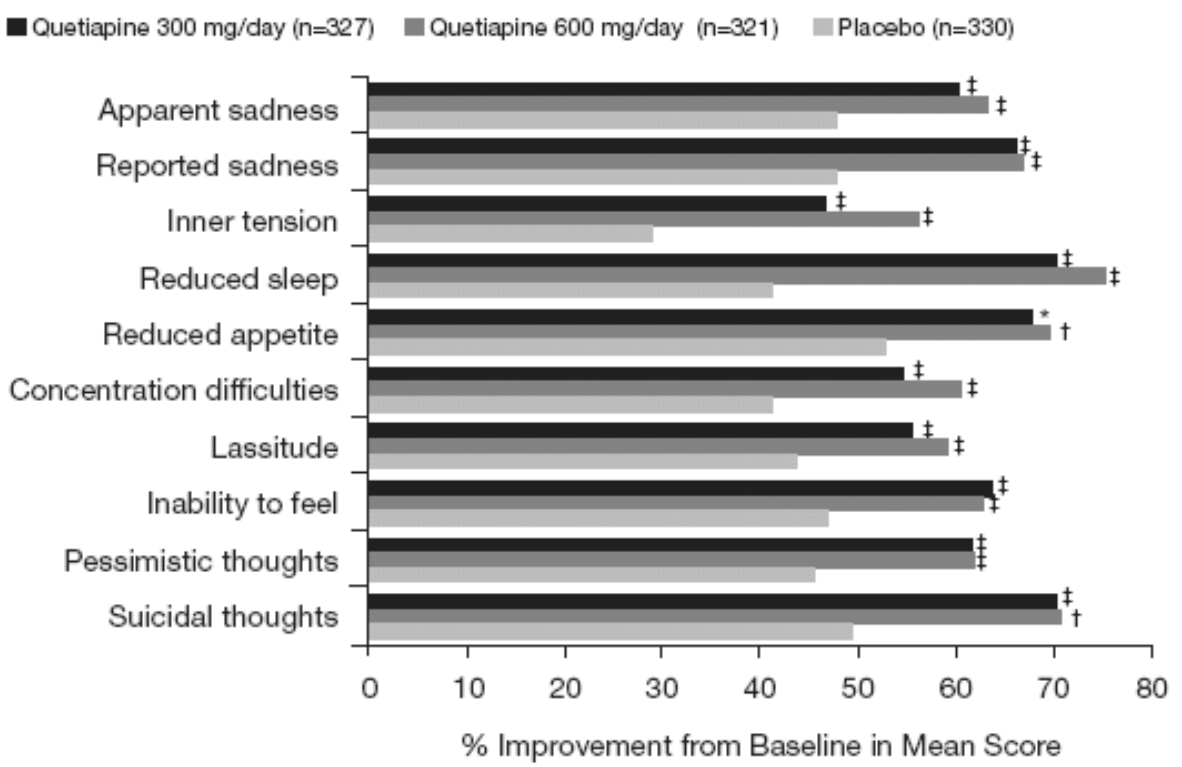

p $<0.05 ;{ }^{\dagger}<<0.01 ;{ }^{\ddagger}<<0.001$ vs placebo

Figure 2 Percentage improvement from baseline in Montgomery-Åsberg Depression Rating Scale (MADRS) individual items scores in outpatients with bipolar I or II disorder (data pooled from BOLDER I and BOLDER II studies; ITT, LOCF).

Rapid cycling is associated with a poorer treatment response and long-term prognosis, and is associated with greater disability and a higher incidence of suicidal behavior (Schneck 2006). Currently available antidepressants may increase the risk of rapid cycling, and this uncertainty has limited their widespread use (Goldberg and Truman 2003). Results of a subanalysis of BOLDER I indicated that quetiapine was as effective in patients with a history of rapid cycling as among with less frequent episodes (Vieta et al 2007). A not yet published analysis of the combined data from the

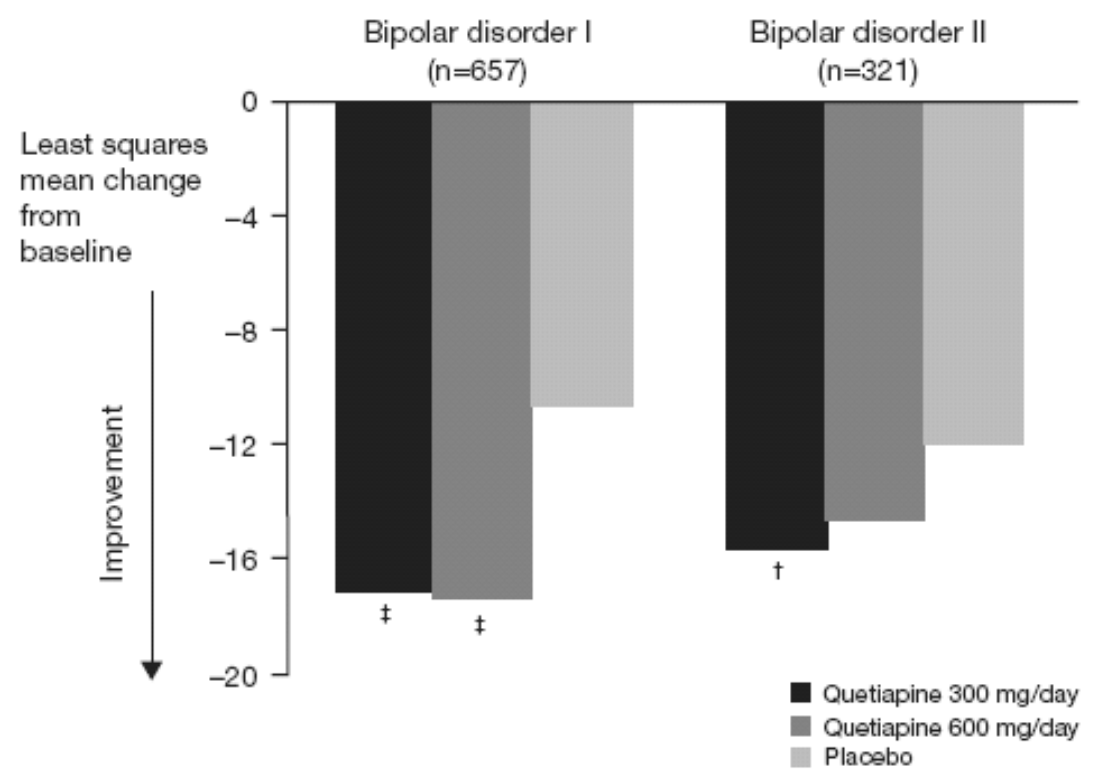

$t_{p<0.01 ;} \ddagger_{p<0}<001$ vs placebo

( $\mathrm{n}$ at baseline)

Figure 3 Least mean squares change from baseline in Montgomery-Åsberg Depression Rating Scale (MADRS) total score in outpatients with bipolar I or II disorder (data pooled from BOLDER I and BOLDER II studies; ITT, LOCF). 
BOLDER studies echoed this result and demonstrated the broad applicability of quetiapine treatment by revealing a similar improvement in MADRS total scores in both patients with or without rapid cycling (Figure 4). As many experts consider antidepressants to be relatively contraindicated in rapid cycling because of an extremely high incidence of TEAS, the antidepressant activity of quetiapine could be partly offset if therapeutic response was associated with increased cycling or the induction of hypomania/mania. It is thus noteworthy that the rapid cycling patients treated with quetiapine in BOLDER I and BOLDER II were no more likely to develop hypomania or mania than were patients receiving placebo. Although patients were also assessed for signs/symptoms of treatment-emergent mood elevation with the Young Mania Rating Scale, to date results on this measure have not been reported. Nevertheless, as described below, the proportion of patients developing diagnosable episodes of hypomania or mania was actually higher among those treated with placebo than those receiving active quetiapine.

\section{Efficacy against anxiety symptoms}

Anxiety disorders are highly comorbid with bipolar disorder and may predispose individuals to intensified symptoms, substance abuse, hospitalization, and suicide ideations (Gaudiano and Miller 2005; Keller 2006;
McIntyre et al 2006). A cross-sectional sample from 500 individuals with bipolar I or II disorder enrolled in the Systematic Treatment Enhancement Program for Bipolar Disorder (STEP-BD) indicated that comorbid anxiety disorders, which were identified in over half of the sample, had a large and negative impact on role functioning and quality of life (Simon et al 2004a). The patient's response to treatment can be dampened by comorbid anxiety (Frank et al 2002; Gaudiano and Miller 2005), making pharmacotherapy a challenge. Despite the high comorbidity, many patients with bipolar disorder and a coexisting anxiety disorder do not receive appropriately tailored drug therapy. In the STEP-BD population mentioned above, only $59 \%$ reported pharmacotherapy use meeting criteria for "minimally adequate" mood stabilizer, regardless of comorbid diagnoses, rapid cycling, or bipolar I or II status (Simon et al 2004b). Moreover, even though anticonvulsants may have some utility for patients with comorbid anxiety, such patients are less responsive to therapy than non-anxious patients (Henry et al 2003). Given the paucity of existing data and the clear need for improved treatment options in this patient subset, it was important to examine the effect of quetiapine monotherapy on anxiety symptoms in the BOLDER studies.

In the BOLDER I study, mixed model for repeated measurements (MMRM) analysis of anxiety symptoms, as

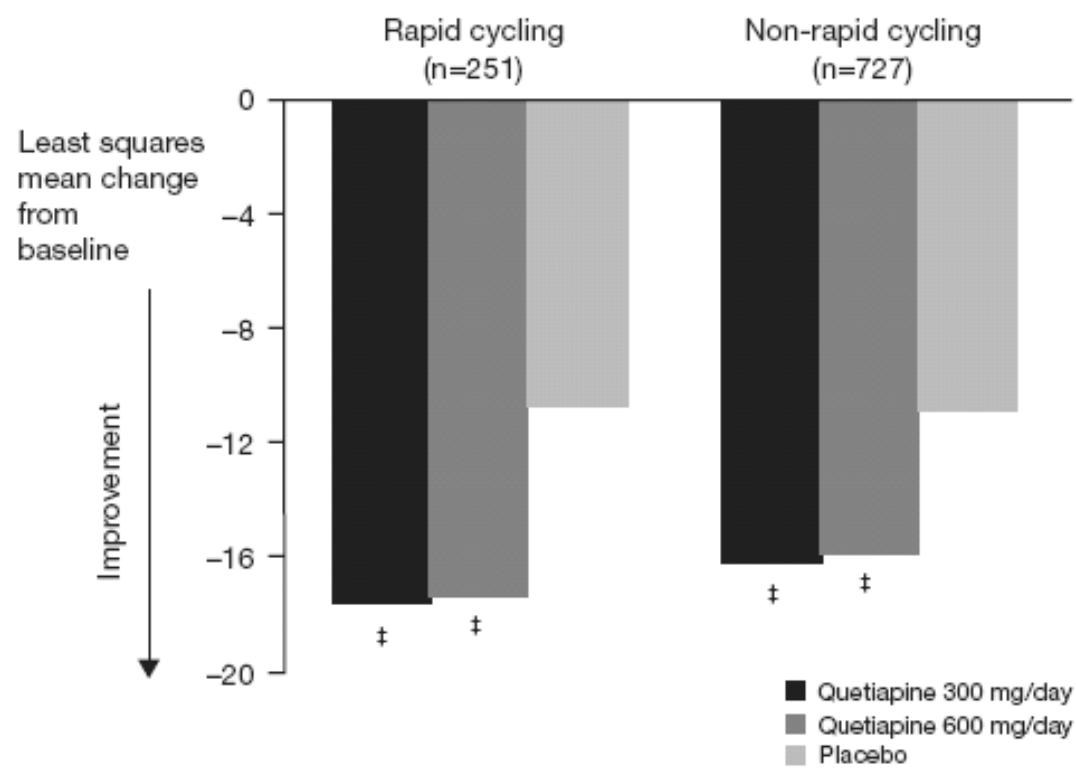

$\ddagger_{\mathrm{p}<0.001}$ vs placebo

( $n$ at baseline)

Figure 4 Least mean squares change from baseline in Montgomery-Åsberg Depression Rating Scale (MADRS) total score in outpatients with bipolar I or II disorder and rapid or non-rapid cycling (data pooled from BOLDER I and BOLDER II studies; ITT, LOCF). 
rated on the Hamilton Rating Scale for Anxiety (HAM-A), revealed that quetiapine monotherapy was associated with a rapid and pronounced improvement across individual and combined quetiapine treatment groups compared with placebo ( -10.3 versus -6.7 for combined quetiapine and placebo groups, respectively), irrespective of baseline severity of depression (Hirschfeld et al 2006). Improvements were seen in eight of the 14 individual items on the HAM-A scale, which included the core items of "anxious mood" $(-1.1$ versus -0.5 , respectively; $p<0.001)$ and "tension" $(-1.1$ versus -0.6 respectively; $\mathrm{p}<0.001)$. This improvement was replicated in the BOLDER II study, in terms of both magnitude and significance (Thase et al 2006).

Improvement in anxiety symptoms was observed in the subgroup of bipolar I patients in BOLDER I, in which HAM-A total score was significantly improved compared with placebo at Week $8(-10.4$ versus -5.1 points, for combined quetiapine and placebo groups, respectively; $\mathrm{p}<0.001$ ) (Hirschfeld et al 2006). Within the smaller subset of patients with bipolar II disorder, patients treated with active quetiapine or placebo showed comparable improvement in HAM-A total score ( -9.8 versus -9.0 , respectively). Results from subgroup analyses from the BOLDER II trial and the pooled data set, not yet available, will be helpful in clarifying this unexpected finding.

\section{Effects on other secondary outcome measures \\ Quality of sleep}

Sleep disturbance is a relatively common occurrence in psychiatric patients, with insomnia presenting as a primary symptom in $30 \%-90 \%$ of psychiatric disorders. (Becker 2006) Again, the BOLDER I study was the first to explore the impact of quetiapine monotherapy on sleep, using the Pittsburgh questionnaire (PSQI) to rate quality of sleep. The PSQI is a 24-item questionnaire that includes 19 self-rated and 5 items rated by the patient's bed partner. The instrument is used to study sleep quality, latency, duration, efficiency, use of medication, and daytime dysfunction, and assesses sleep quality and disturbance in the preceding month.

In BOLDER I, participants showed moderate-to-severe sleep disruption at baseline according to the PSQI. At last assessment, quality of sleep had improved significantly following treatment with either dose of quetiapine compared with placebo ( $p<0.001)$ (Endicott et al 2007). Mean values for quality of sleep, sleep latency, and sleep duration were $0.5-0.7$ points lower in quetiapine-treated patients than in placebo-treated patients (Endicott et al 2007).

\section{Quality of life}

To date, dedicated quality-of-life studies in individuals with bipolar disorder are relatively sparse, although a number of ongoing studies are attempting to redress this imbalance in response to increased recognition that recovery should be marked by a return to an acceptable quality of life and improved functionality for the patient (Michalak et al 2005; Mitchell et al 2006; Kasper 2004). The depressive component of bipolar disorder is thought to be more detrimental to quality of life than the manic component (Vojta et al 2001). Furthermore, the degree of functional impairment and loss of work productivity experienced by individuals with bipolar depression exceeds that experienced by patients with unipolar depression (Vornik and Hirschfeld 2005). There is also evidence to suggest that the delayed diagnosis or misdiagnosis experienced by many individuals with bipolar depression may further impact the quality of life they are able to enjoy since early and appropriate intervention can drastically enhance not only the symptomatic but also the subjective experience of individuals over the long term (Kasper 2004).

Thus, any efficacious medication that can also exert positive effects on quality of life in bipolar depression is of obvious therapeutic value. Positive effects on health-related quality of life may be intrinsically linked with general tolerability. If a treatment is well-tolerated, a patient will be more likely to become satisfied with his treatment regimen. The superiority and increasing popularity of the atypical antipsychotics over the more conventional antipsychotics may derive from their improved tolerability (Vornik and Hirschfeld 2005).

The BOLDER I study was the first large-scale investigation of quetiapine monotherapy in bipolar depression to report quality of life as a secondary endpoint (Endicott et al 2007). Both BOLDER studies used the short-form version of the Quality of Life Enjoyment and Satisfaction Questionnaire (Q-LES-Q) to evaluate the impact of quetiapine on healthrelated quality of life. This version rates 16 items (physical health; mood; work; household activities; social relationships; family relationships; leisure-time activity; ability to function in daily life; sexual drive, interest and/or performance; economic status; living/housing situation; ability to get around physically without feeling dizzy or unsteady or falling; vision in terms of ability to work or do hobbies; overall sense of well-being; medications; and overall life satisfaction and contentment during the past week) on a 5-point scale.

Consistent with their disease profile, BOLDER participants scored poorly on quality-of-life scores at baseline. 
Following 8 weeks of treatment with quetiapine, significant improvements in Q-LES-Q total score were evident in both BOLDER studies (Calabrese et al 2005; Thase et al 2006; Endicott et al 2007). In BOLDER I, for example, mean Q-LES-Q percentage maximum scores at final assessment (LOCF) were 57.6, 57.4, and 48.1 points for $300 \mathrm{mg}$ quetiapine, $600 \mathrm{mg}$ quetiapine, and placebo, respectively (Endicott et al 2007). The improvements were significantly greater in both quetiapine groups compared with placebo $(\mathrm{p}<0.001)$ at final assessment. Interestingly, quality-oflife improvements were positively linked with MADRS response and remission status. If a patient responded to quetiapine treatment, quality of life also improved, whereas MADRS non-response was associated with negligible improvement in quality of life.

The Sheehan Disability Scale (SDS), introduced as an additional outcome measure for BOLDER II, quantified the impact of quetiapine on the interrelated domains of work, social life, and family life/home life/responsibilities. At the last assessment, the mean improvements in SDS scores from baseline were $-7.3,-7.9$, and -6.0 for $300 \mathrm{mg}$ quetiapine, $600 \mathrm{mg}$ quetiapine, and placebo, respectively ( $\mathrm{p}<0.05$ for $600 \mathrm{mg}$ quetiapine versus placebo) (Thase et al 2006).

\section{Tolerability profile}

Good tolerability, as rated by physicians and patients alike, is integral to the success of any medication strategy. As tolerability and treatment adherence tend to worsen as each new drug is added to a complex pharmacotherapy regimen, a welltolerated monotherapy would be expected to have multiple advantages. (Burton et al 2005) Quetiapine monotherapy was generally well-tolerated in the BOLDER studies. As detailed safety/tolerability data were presented in the source publications, (Calabrese et al 2005; Thase et al 2006) only a brief summary follows below.

\section{Treatment discontinuations and adverse event $(A E)$ incidence}

In the BOLDER studies, the proportions of quetiapine-treated patients completing the full 8 week treatment protocol was similar to that reported for placebo and ranged between 53\% and $67 \%$. The most common reasons for discontinuation were withdrawal of consent, adverse events, loss to follow-up, and lack of perceived efficacy, with attrition due to AEs more common among the patients receiving active medication and dropouts due to lack of efficacy more common in the placebo groups. None of the serious AEs reported in either BOLDER study was considered to have been drug-related.
The majority of AEs were mild-to-moderate in intensity and transient in nature. The most common adverse events experienced by $5 \%$ or more of patients receiving quetiapine in both BOLDER studies were dry mouth, sedation, somnolence, dizziness, and constipation (Table 1; pooled data from BOLDER I and II). Trends in tolerability tended to favor the group receiving the lower (300 mg per day) dose, although few of these differences were statistically significant.

\section{Weight change}

Weight gain in the BOLDER studies was greater among the patients receiving active quetiapine. Across studies, the pooled weight-gain data showed a mean increase of $1.2 \mathrm{~kg}$ in the quetiapine $300 \mathrm{mg}$ per day group and $1.5 \mathrm{~kg}$ in the $600 \mathrm{mg}$ per day group, as compared with a gain of $0.2 \mathrm{~kg}$ in the placebo group. A weight increase of greater than $7 \%$ was observed in $7.1 \%$ of patients receiving the $300 \mathrm{mg} /$ day dose of quetiapine, $10.0 \%$ receiving the $600 \mathrm{mg} /$ day dose of quetiapine, and $2.4 \%$ of those receiving placebo.

The weight gain associated with some atypical antipsychotics may often be accompanied by deleterious changes in serum lipids and increases in fasting glucose, both of which increase the risk for coronary artery disease. It is important to note that in the BOLDER trials, the mean change in fasting glucose and lipids with quetiapine was not statistically significantly different to that with placebo.

\section{Treatment-emergent mania}

As discussed previously, the risk of precipitating a manic episode is a major concern when treating bipolar depression with traditional antidepressants. Treatment-emergent mania affects $20 \%-40 \%$ of individuals with bipolar disorder receiving long-term antidepressant therapy (Goldberg and Truman 2003). Since combination therapy may reduce the risk of treatment-emergent mania, this is often the treatment approach

Table I Most common adverse events associated with quetiapine treatment in outpatients with bipolar I or II disorder ( $\geq 5 \%$ patients; data pooled from BOLDER I and BOLDER II studies)

\begin{tabular}{lccc}
\hline $\begin{array}{l}\text { Adverse } \\
\text { event (\%) }\end{array}$ & $\begin{array}{c}\text { Quetiapine } \\
\mathbf{3 0 0} \mathbf{~} \mathbf{g} \\
(\mathbf{n = 3 5 0 )}\end{array}$ & $\begin{array}{l}\text { Quetiapine } \\
\mathbf{6 0 0} \mathbf{~} \mathbf{3} \\
(\mathbf{n = 3 4 8})\end{array}$ & $\begin{array}{c}\text { Placebo } \\
(\mathbf{n}=\mathbf{3 4 7})\end{array}$ \\
\hline Dry mouth & 43.4 & 43.7 & 12.7 \\
Sedation & 30.9 & 29.9 & 8.1 \\
Somnolence & 28.6 & 27.0 & 6.6 \\
Dizziness & 15.4 & 19.5 & 6.9 \\
Constipation & 10.0 & 10.6 & 3.7 \\
Lethargy & 5.7 & 5.2 & 1.7 \\
Weight increase & 2.9 & 5.7 & 1.2 \\
\hline
\end{tabular}


preferred by many psychiatrists (Grunze 2005). Therefore, when assessing the tolerability profile of a single agent, it is important to establish the propensity for manic switch.

Data from the BOLDER studies showed a low incidence of treatment-emergent mania with quetiapine and placebo. Treatment-emergent mania was defined as an adverse event of mania or hypomania, or Young Mania Rating Scale (YMRS) score $\geq 16$ on any two consecutive visits or at the final visit. Data pooled from BOLDER I and II reveal that the proportion of patients with treatment-emergent manic symptoms was greater in the placebo group (5.2\%) compared with both quetiapine treatment groups (both $2.9 \%$ ).

\section{EPS-related AEs}

Historically, the atypical antipsychotics have been associated with a reduced risk of extrapyramidal symptoms (EPS) within the recommended dose ranges compared with their conventional counterparts (Pierre 2005). This highly desirable characteristic is thought to derive directly from their mechanism of action, specifically by less avid binding to post-synaptic dopamine receptors in the basal ganglia (Seeman 2002; Tort et al 2006).

In the BOLDER trials, no individual specific EPS had an incidence greater than $5 \%$ among patients receiving quetiapine and the rate of discontinuation due to EPS was also low. The incidence of the abnormal involuntary movements that were considered to be EPS was $12.0 \%$ with quetiapine $300 \mathrm{mg}$ per day, $11.5 \%$ with quetiapine $600 \mathrm{mg}$ per day, and $5.5 \%$ with placebo. Mean changes in scores from rating scales used to assess EPS, the Simpson-Angus Scale and the Barnes Akathisia Rating Scale, were low in the quetiapine treatment groups and were similar to those reported for placebo.

\section{Long-term efficacy}

One significant limitation of the BOLDER studies is that they were limited to only 8 weeks of double-blind therapy and patients were withdrawn from study medication at the completion of the study. In clinical practice, an effective monotherapy of bipolar depression would typically be maintained indefinitely for prophylaxis. To date, the long-term effects of quetiapine in bipolar depression have only been assessed in a small open-label study (Milev et al 2006), and with quetiapine (at doses up to $800 \mathrm{mg} /$ day) being used in combination with ongoing antidepressant or mood stabilizing therapy. A naturalistic 12-month study in 17 patients revealed a mean reduction in baseline HAM-D total score of $55.5 \%$ LOCF following the addition of quetiapine to the existing treatment regimen (Milev et al 2006). The proportion of patients $(n=13)$ achieving a $50 \%$ reduction in
HAM-D ${ }_{17}$ total score was high, at $76.5 \%$. Although these preliminary data are encouraging, larger controlled studies are required to extend these long-term findings and to examine the long-term effects of quetiapine monotherapy in particular. Maintenance studies with quetiapine in bipolar disorder are currently under analysis. The impact of longer term quetiapine therapy on weight and other metabolic indices will be of considerable interest.

\section{Relative efficacy}

Another limitation of the BOLDER studies is that neither study included an active comparator. Now that efficacy has been established, comparative studies are needed, both versus conventional mood stabilizers such as lithium and valproate, and the olanzapine - fluoxetine combination (OFC) (the only other treatment strategy now approved for treatment of bipolar depression). Some would argue that head-to-head comparisons versus lamotrigine also are needed. Although the acute phase efficacy of lamotrigine has not yet been established by a series of unequivocally positive RCTs, it is widely used by clinicians for treatment of both bipolar I and bipolar II depression and there is, at the least, evidence suggestive of efficacy in the literature (Calabrese et al 1999; Frye et al 2000). In the only study to directly compare lamotrigine and OFC, trends favoured the latter drug on efficacy measures, although tolerability indices clearly favoured lamotrigine (Brown et al 2006). In addition to studies of quetiapine as a monotherapy, its utility in combination with other relevant therapies, both antidepressants and conventional mood stabilizers, warrant evaluation.

\section{Is quetiapine a mood stabilizer?}

There is currently a lack of consensus surrounding the label "mood stabilizer," but generally, if an agent shows efficacy in treating both acute manic and depressive symptoms and is also effective in the prevention of recurrences, it can be considered as such (Bauer and Mitchner 2004; Goodwin and Malhi 2007). Currently, no one treatment adequately fulfills the "ideal" mood stabilizer criteria, although the popular view is that lithium comes closest to doing so (Sachs 2005). The emergence of quetiapine, with its bimodal activity against bipolar mania and depression, may qualify as a mood stabilizer in its own right (Vieta 2005; Sachs 2005).

The defining criteria for a mood stabilizer mentioned earlier omit other desirable characteristics like good tolerability, which tend to become more relevant once efficacy has been established (Bauer and Mitchner 2004). Clearly, if a treatment is well tolerated, then adherence, quality of life, and caregiver 
benefits will also improve. So if the antidepressant efficacy of quetiapine derived from BOLDER and its proven ability in improving manic symptoms from earlier studies place quetiapine monotherapy in a strong position as a potential mood stabilizer, its tolerability profile is favorable enough to permit first line use if syndromal severity is sufficient to justify prescription of an atypical antipsychotic. The low rate of treatment-emergent mania gives quetiapine an obvious advantage over the combination of a mood stabilizer and a traditional antidepressant. Nevertheless, the strong performance of quetiapine in patients with a history of rapid cycling observed in the BOLDER studies does warrant prospective replication before this medication can truly be considered a treatment of choice for this clinically challenging presentation of bipolar disorder. Although longer-term data in bipolar disorder are needed, results of the BOLDER studies are reassuring that a large majority of patients will not gain a meaningful amount of weight during acute-phase therapy. Accompanying quetiapine-induced improvements in quality of sleep, functionality, and health-related quality of life will impact not only the patient, but the family members or caregivers who surround them. Ultimately, caregiver perception and not just patient perception will dictate the success of quetiapine as a mood-stabilizing treatment option. Dedicated studies in this area are awaited with interest.

\section{Conclusions}

The BOLDER studies demonstrated that two doses of quetiapine monotherapy - $300 \mathrm{mg}$ and $600 \mathrm{mg}$ - prescribed at bedtime were rapidly effective and generally well tolerated in bipolar depression. The antidepressant efficacy of quetiapine was found in both bipolar I and bipolar II depression and extended to patients with high levels of anxiety as well as those with a history of rapid cycling. Quetiapine was also associated with significant improvements in health-related quality of life, improvements that were intrinsically linked to its antidepressant efficacy. Comparative trials are now needed to help to rank quetiapine therapy among the group of other widely regarded first-line options, and long-term data from controlled studies of patients treated for bipolar depression are also needed to ensure that these effects are durable and that tolerability is acceptable across months or even years of therapy.

\section{Acknowledgments and Disclosures}

The author would like to acknowledge the editorial assistance of Eleanor Bull, PhD (PAREXEL MMS). Financial support for this assistance was provided by AstraZeneca Pharmaceuticals LP.

\section{References}

AstraZeneca. Seroquel ${ }^{\circledR}$ (quetiapine fumarate) tablets: prescribing information [online]. Accessed 2007 April 18. URL: http://www.seroquel.com

Bauer MS, Mitchner L. 2004. What is a "mood stabilizer"? An evidencebased response. Am J Psychiatry, 161:3-18.

Becker PM. 2006. Treatment of sleep dysfunction and psychiatric disorders. Curr Treat Options Neurol, 8:367-75.

Brown EB, McElroy SL, Keck PE Jr, et al. 2006. A 7-week, randomized, double-blind trial of olanzapine/fluoxetine combination versus lamotrigine in the treatment of bipolar I depression. J Clin Psychiatry, 67:1025-33.

Burton SC. 2005. Strategies for improving adherence to second-generation antipsychotics in patients with schizophrenia by increasing ease of use. J Psychiatr Pract, 11:369-78.

Calabrese JR, Bowden CL, Sachs GS, et al. 1999. A double-blind placebocontrolled study of lamotrigine monotherapy in outpatients with bipolar I depression. Lamictal 602 Study Group. J Clin Psychiatry, 60:79-88.

Calabrese JR, Keck PE Jr, Macfadden W, et al. 2005. A randomized, doubleblind, placebo-controlled trial of quetiapine in the treatment of bipolar I or II depression. Am J Psychiatry, 162:1351-60.

Depp CA, Davis CE, Mittal D, et al. 2006. Health-related quality of life and functioning of middle-aged and elderly adults with bipolar disorder. J Clin Psychiatry, 67:215-21.

Dilsaver SC, Chen YW, Swann AC, et al. 1997. Suicidality, panic disorder and psychosis in bipolar depression, depressive-mania and pure-mania. Psychiatry Res, 73:47-56.

Endicott J, Rajagopalan K, Minkwitz M, et al. 2007. A randomized, double-blind, placebo-controlled study of quetiapine in the treatment of bipolar I and II depression: improvements in quality of life. Int Clin Psychopharmacol, 22:29-37.

Fagiolini A, Frank E, Scott JA, et al. 2005. Metabolic syndrome in bipolar disorder: findings from the Bipolar Disorder Center for Pennsylvanians. Bipolar Disord, 7:424-30.

Frank E, Cyranowski JM, Rucci P, et al. 2002. Clinical significance of lifetime panic spectrum symptoms in the treatment of patients with bipolar I disorder. Arch Gen Psychiatry, 59:905-11.

Frye MA, Ketter TA, Kimbrell TA, et al. 2000. A placebo-controlled study of lamotrigine and gabapentin monotherapy in refractory mood disorders. J Clin Psychopharmacol, 20:607-14.

Gaudiano BA, Miller IW. 2005. Anxiety disorder comobidity in Bipolar I Disorder: relationship to depression severity and treatment outcome. Depress Anxiety, 21:71-7.

Goldberg JF, Truman CJ. 2003. Antidepressant-induced mania: an overview of current controversies. Bipolar Disord, 5:407-20.

Goodwin GM, Malhi GS. 2007. What is a mood stabilizer? Psychol Med, In Press.

Grunze H. 2005. Reevaluating therapies for bipolar depression. J Clin Psychiatry, 66(Suppl 5):17-25.

Henry C, Van den Bulke D, Bellivier F, et al. 2003. Anxiety disorders in 318 bipolar patients: prevalence and impact on illness severity and response to mood stabilizer. J Clin Psychiatry, 64:331-5.

Hirschfeld RM. 2004. Bipolar depression: the real challenge. Eur Neuropsychopharmacol, 14(Suppl 2):S83-8.

Hirschfeld RM, Weisler RH, Raines SR, et al. 2006. Quetiapine in the treatment of anxiety in patients with bipolar I or II depression: a secondary analysis from a randomized, double-blind, placebo-controlled study. J Clin Psychiatry, 67:355-62.

Judd LL, Akiskal HS, Schettler PJ, et al. 2002. The long-term natural history of the weekly symptomatic status of bipolar I disorder. Arch Gen Psychiatry, 59:530-7.

Judd LL, Akiskal HS, Schettler PJ, et al. 2003. A prospective investigation of the natural history of the long-term weekly symptomatic status of bipolar II disorder. Arch Gen Psychiatry, 60:261-9.

Judd LL, Akiskal HS, Schettler PJ, et al. 2005. Psychosocial disability in the course of bipolar I and II disorders: a prospective, comparative, longitudinal study. Arch Gen Psychiatry, 62:1322-30. 
Kasper SF. 2004. Living with bipolar disorder. Expert Rev Neurother, 4; 6(Suppl 2):S9-15.

Keck PE Jr. 2005. Bipolar depression: a new role for atypical antipsychotics? Bipolar Disord, 7(Suppl 4):34-40.

Keller MB. 2006. Prevalence and impact of comorbid anxiety and bipolar disorder. J Clin Psychiatry, 67(Suppl 1):5-7.

Ketter TA, Calabrese JR. 2002. Stabilization of mood from below versus above baseline in bipolar disorder: a new nomenclature. J Clin Psychiatry, 63:146-51.

McIntyre RS, Soczynska JK, Bottas A, et al. 2006. Anxiety disorders and bipolar disorder: a review. Bipolar Disord, 8:665-76.

Michalak EE, Yatham LN, Lam RW. 2005. Quality of life in bipolar disorder: a review of the literature. Health Qual Life Outcomes, 3:72.

Milev R, Abraham G, Zaheer J. 2006. Add-on quetiapine for bipolar depression: a 12-month open-label trial. Can J Psychiatry, 51:523-30.

Mitchell PB, Malhi GS. 2004. Bipolar depression: phenomenological overview and clinical characteristics. Bipolar Disord, 6:530-9.

Mitchell PB, Ball JR, Best JA, et al. 2006. The management of bipolar disorder in general practice. Med J Aust, 184:566-70.

Muller MJ, Himmerich H, Kienzle B, et al. 2003. Differentiating moderate and severe depression using the Montgomery-Asberg depression rating scale (MADRS). J Affect Disord, 77:255-60.

Murray CJ, Lopez AD. 1997. Regional patterns of disability-free life expectancy and disability-adjusted life expectancy: global Burden of Disease Study. Lancet, 349:1347-52.

Nemeroff CB. 2005. Use of atypical antipsychotics in refractory depression and anxiety. J Clin Psychiatry, 66(Suppl 8):13-21.

Ogilvie AD, Morant N, Goodwin GM. 2005. The burden on informal caregivers of people with bipolar disorder. Bipolar Disord, 7(Suppl 1):25-32.

Ostacher MJ. 2006. The evidence for antidepressant use in bipolar depression. J Clin Psychiatry, 67(Suppl 11):18-21

Perlick DA, Rosenheck RA, Clarkin JF, et al. 2004. Impact of family burden and affective response on clinical outcome among patients with bipolar disorder. Psychiatr Serv, 55:1029-35.

Pierre JM. 2005. Extrapyramidal symptoms with atypical antipsychotics : incidence, prevention and management. Drug Saf, 28:191-208.

Post RM. 2005. The impact of bipolar depression. J Clin Psychiatry, 66(Suppl 5):5-10.

Post RM, Denicoff KD, Leverich GS, et al. 2003. Morbidity in 258 bipolar outpatients followed for 1 year with daily prospective ratings on the NIMH life chart method. J Clin Psychiatry, 64:680-90.

Sachs GS. 2005. What is a mood stabilizer? Clin Appr Bipolar Disord, 4:3.

Sachs GS, Nierenberg AA, Calabrese JR, et al. 2007. Effectiveness of adjunctive antidepressant treatment for bipolar depression. $N$ Engl $J$ Med, 356:1711-22.
Schneck CD. 2006. Treatment of rapid-cycling bipolar disorder. $J$ Clin Psychiatry, 67(Suppl 11):22-7.

Seeman P. 2002. Atypical antipsychotics: mechanism of action. Can $J$ Psychiatry, 47:27-38.

Simon NM, Otto MW, Wisniewski SR, et al. 2004a. Anxiety disorder comorbidity in bipolar disorder patients: data from the first 500 participants in the Systematic Treatment Enhancement Program for Bipolar Disorder (STEP-BD). Am J Psychiatry, 161:2222-9.

Simon NM, Otto MW, Weiss RD, et al. 2004b. Pharmacotherapy for bipolar disorder and comorbid conditions: baseline data from STEP-BD. J Clin Psychopharmacol, 24:512-20.

Thase ME. 2005. Bipolar depression: issues in diagnosis and treatment. Harv Rev Psychiatry, 13:257-71.

Thase ME, Macfadden W, Weisler RH, et al. 2006. Efficacy of quetiapine monotherapy in bipolar I and II depression: a double-blind, placebocontrolled study (the BOLDER II study). J Clin Psychopharmacol, 26:600-9.

Tohen M, Vieta E, Calabrese J, et al. 2003. Efficacy of olanzapine and olanzapine-fluoxetine combination in the treatment of bipolar I depression. Arch Gen Psychiatry, 60:1079-88.

Tort AB, Souza DO, Lara DR. 2006. Theoretical insights into the mechanism of action of atypical antipsychotics. Prog Neuropsychopharmacol Biol Psychiatry, 30:541-8.

Vieta E. 2005. Mood stabilization in the treatment of bipolar disorder: focus on quetiapine. Hum Psychopharmacol, 20:225-36.

Vieta E, Calabrese J, Goikolea J, et al; for the BOLDER Study Group. 2007. Quetiapine monotherapy in the treatment of patients with bipolar I or II depression and a rapid-cycling disease course: a randomized, doubleblind, placebo-controlled study. Bipolar Disord, 9:413-25.

Vojta C, Kinosian B, Glick H, et al. 2001. Self-reported quality of life across mood states in bipolar disorder. Compr Psychiatry, 42:190-5.

Vornik LA, Hirschfeld RM. 2005. Bipolar disorder: quality of life and the impact of atypical antipsychotics. Am J Manag Care, 11(Suppl 9): S275-80.

Yatham LN, Kennedy SH, O'Donovan C, et al. 2005. Canadian Network for Mood and Anxiety Treatments (CANMAT) guidelines for the management of patients with bipolar disorder: consensus and controversies. Bipolar Disord, 7(Suppl 3):5-69.

Yatham LN, Kennedy SH, O'Donovan C, et al. 2006. Canadian Network for Mood and Anxiety Treatments (CANMAT) guidelines for the management of patients with bipolar disorder: update 2007. Bipolar Disord, 8:721-39.

Zornberg GL, Pope HG Jr, 1993. Treatment of depression in bipolar disorder: new directions for research. J Clin Psychopharmacol, 13:397-408. 
